\title{
The full life revisited: Examining hedonia and eudaimonia as general orientations, motives for activities, and experiences of wellbeing
}

\author{
Taysa-Rhea Mise · Michael A. Busseri
}

\begin{abstract}
We examined the concepts of hedonia and eudaimonia with respect to general orientations to life, motives for activities, and experiences of wellbeing among young Canadian adults $(N=325 ; M$ age $=19.10, S D=2.18 ; 87 \%$ female $)$. Within concepts, moderate positive correlations were observed across facets of hedonia and eudaimonia. Between concepts, correlations were strongest for wellbeing and weakest for motives. Unique predictive associations of eudaimonic general orientations and motives for daily activities were found for both types of wellbeing. In support of the 'full life' hypothesis the highest levels of each form of wellbeing were found among individuals characterized by high (top 33\%) levels of hedonic and eudaimonic general orientations and motivations for recent activities. Findings support distinguishing between hedonia and eudaimonia as related but independent concepts, and provide new insights concerning hedonic and eudaimonic general orientations and motivations for activities in characterizing the full life.
\end{abstract}

Keywords: hedonia, eudaimonia, orientations, motives, wellbeing

\section{Introduction}

Psychological research on the concept of 'wellbeing' typically focuses on experiences of 'hedonia' - usually studied in terms of satisfaction and enjoyment of one's life, or 'eudaimonia' - which encompasses various aspects of positive functioning, including personal growth, authenticity, and meaning in life (Ryan \& Deci, 2001; Waterman, 1993). Hedonia and eudaimonia apply not just to experiences of wellbeing, but also to how individuals generally orient their lives and their motives for the activities in which they engage (Huta \& Waterman, 2014; Peterson, Park, \& Seligman, 2005). Understanding the similarities and differences between these concepts is a central issue in research on positive psychology. To advance this issue, the present study examined associations between hedonia and eudaimonia with respect to general orientations to life, motives for daily activities, and experiences of wellbeing. Furthermore, we evaluated the combined and unique predictive effects of hedonic and eudaimonic general orientations and motives for activities on hedonic and eudaimonic wellbeing.

Psychological research on wellbeing is dominated by two traditions: hedonia and eudaimonia (Ryan \& Deci 2001; Waterman, 1993). Hedonic wellbeing is most often assessed as 'subjective wellbeing' (Diener, 1984) and is typically studied based on three components: a subjective appraisal of satisfaction with one's life overall, the presence of positive affect, and the absence of negative affect (Busseri \& Sadava, 2011; Diener, Suh, Lucas, \& Smith, 1999). Various operationalizations of eudaimonic wellbeing have been proposed (Huta \& Waterman, 2014), but it is often studied with respect to indicators of positive functioning including: positive 
relationships, personal growth, life purpose, mastery, autonomy, and self-acceptance (Ryff, 1989); as well as experiences of meaning, authenticity, and personal expressiveness (Waterman, Schwartz, Zamboanga, et al., 2010). Although conceptually distinct, self-report measures of hedonic and eudaimonic wellbeing are typically strongly and positively correlated (Gallagher, Lopez, \& Preacher, 2009; Joshanloo, 2016; Keyes, Shmotkin, \& Ryff, 2002) leading some to question the validity (or value) of differentiating these two forms of wellbeing (Kashdan, BiswasDiener, \& King, 2008). Nonetheless, there remains strong interest in understanding the similarities and differences between hedonia and eudaimonia (Huta \& Waterman, 2014), not only in terms of experiences of wellbeing, but also with respect to how individuals live their lives (Ryan \& Huta, 2009), including their general orientations to life (Peterson et al., 2005) and motives for personal activities (Huta \& Ryan, 2010).

General orientations refer to individuals' underlying motives and goals for their lives, and are important contributors to wellbeing (Diener \& Lucas 1999; Peterson et al., 2005; Ryan \& Deci 2001). Although such tendencies guide behavior, situational constraints and immediate needs may also impact decisions concerning daily activities. Thus, attention has been given to the types of motives that individuals pursue in their daily activities. Whereas hedonically oriented individuals seek regular pleasure and enjoyment, eudaimonically oriented individuals seek meaningful and authentic experiences to becoming their best selves (Huta \& Waterman, 2014; Peterson et al., 2005). In contrast to the strong positive correlation typically observed between hedonic and eudaimonic wellbeing, hedonia and eudaimonia as motives for activities may share considerably less overlap (Huta, 2013; Huta \& Waterman, 2014).

Furthermore, hedonic and eudaimonic motives may be related to similar as well as distinct aspects of hedonic and eudaimonic wellbeing (Asano, Tsukamoto, Igarashi, \& Huta, 2018; Huta, 2016; Huta, Pelletier, Baxtor, \& Thompson, 2012; Huta \& Ryan, 2010). In particular, some studies suggest that whereas hedonic motives may be linked with various aspects of hedonic wellbeing, such motives may not be uniquely related to indicators of eudaimonic wellbeing, controlling for eudaimonic motives. Such findings suggest that hedonic motives such as seeking pleasure may be uniquely aligned with (or predictive of) hedonic wellbeing, rather than supportive of eudaimonic aspects of wellbeing such as personal growth and authenticity. In addition, whereas eudaimonic motives may be linked with eudaimonic wellbeing, such motives may not be uniquely related to hedonic wellbeing, controlling for eudaimonic motives (e.g. McGregor \& Little, 1998; Steger, Kashdan, \& Oishi, 2008). Such findings suggest that eudaimonic motives such as seeking meaning and purpose in one's life may be uniquely aligned with (or predictive of) eudaimonic wellbeing, rather than supportive of hedonic aspects of wellbeing such as enjoyment and pleasure. Other research suggests that eudaimonic motives for activities may contribute uniquely to both forms of wellbeing (e.g., Huta et al., 2012). It is possible, therefore, that seeking meaning and excellence in one's activities facilitates personal growth and authentic living as well as satisfaction and enjoyment (Huta \& Waterman, 2014; Waterman, 1993, Waterman, Schwartz, \& Conti, 2008).

If so, hedonic and eudaimonic motives may work in complimentary, rather than opposing ways to promote a wide range of positive life outcomes (Huta \& Waterman, 2014; Ryan \& Huta, 2009). In support of this 'full life' notion (Seligman, 2002), some studies suggest that the most positive outcomes (including wellbeing, psychological functioning, and academic success) are found among individuals reporting higher levels of both hedonic and eudaimonic orientations or motives. Importantly, research on the full life has focused either on general orientations to life (Peterson et al., 2005; Schueller \& Seligman, 2010) or motives for activities (Huta \& Ryan, 2010; Kryza-Lacombe, Tanzini, \& O'Neill, 2019), but not both. Yet to be determined is how hedonic 
and eudaimonic general orientations towards one's life are related to hedonic and eudaimonic motives for activities, and how each of these relates to hedonic and eudaimonic wellbeing.

\subsection{The present study}

In this study our aim was to further the understanding of hedonia and eudaimonia with respect to individuals' general orientations towards their lives, motives for daily activities, and overall wellbeing. We predicted positive associations between each facet of hedonia (i.e., general orientations, motives for daily activities, wellbeing) and between each facet of eudaimonia. We also expected positive associations between each corresponding facet of hedonia and eudaimonia (e.g., hedonic and eudaimonic general orientations), but that such linkages would be stronger with respect to wellbeing than daily activity motives (Huta \& Waterman, 2014). To inform the combined and unique predictive effects, we also examined general orientations and motives for daily activities as joint predictors of hedonic and eudaimonic wellbeing, expecting positive correlations between each predictor and both forms of wellbeing (Huta, 2016). We also expected the unique predictive effects of hedonic orientations and motives for activities to be limited to hedonic (rather than eudaimonic) wellbeing, whereas eudaimonic orientations and motives would uniquely contribute to the prediction of both forms of wellbeing (Huta et al., 2012). Finally, we evaluated the full life hypothesis (Huta \& Ryan, 2010; Peterson et al., 2005) based on consideration of general orientations and motives for daily activities. We expected that the highest levels of hedonic and eudaimonic wellbeing would be found among individuals characterized by high levels of hedonic and eudaimonic general orientations and high levels of hedonic and eudaimonic motives for daily activities.

\section{Method}

\subsection{Participants and procedure}

Canadian undergraduate students from Brock University, Canada $(N=334 ; M$ age $=19.14, S D=$ 2.22; 87\% female; academic majors included Psychology, 31\%, Child and Youth Studies, 17\%, and Education, $11 \%$ ) completed a questionnaire in small-group settings in return for course credit. Nine participants were removed due to incomplete data (i.e., less than $50 \%$ of the data for at least one of the analysis variables described below). The final analysis sample thus comprised 325 participants $(M$ age $=19.10, S D=2.18 ; 87 \%$ female $)$.

\subsection{Measures}

\subsubsection{Hedonic and eudaimonic general orientations and motives for daily activities}

The Hedonic and Eudaimonic Motives for Activities was developed by Huta and Ryan (2010) to assess individuals" motives for their activities, using the following prompt: "To what extent do you typically approach your activities with the following intentions, whether or not you actually achieve your aim?". The nine-item scale comprises five hedonic (e.g., "Seeking pleasure") and four eudaimonic (e.g., "Seeking to use the best in yourself") items, each rated on a seven-point scale $(1=$ not at all and $7=$ very $m u c h)$. Wording for all nine items is provided in Huta \& Ryan (2010, Table 1). Ratings for the hedonic and eudaimonic items are averaged separately. This scale was modified for the present purpose to measure the extent to which individuals (1) had a general orientation toward hedonic and eudaimonic aims in life, and (2) had hedonic and eudaimonic motives for their recent daily lives. To do so, participants completed the scale twice, the first time in response to the following prompt: "In general, how important is each of the following to you in your life?" Ratings were averaged separately for the hedonic and eudaimonic 
items ( $\alpha \mathrm{s}=.75$ and .74) such that higher scores indicated stronger hedonic and eudaimonic general orientations toward one's life, respectively. On a separate page, participants completed the scale a second time in response to the following prompt: "During the past week, to what degree did you approach your activities with each of the following intentions, whether or not you actually achieved your aim?" Ratings were averaged such that higher scores indicated stronger hedonic and eudaimonic motives for recent daily activities, respectively $(\alpha s=.80$ and $.78)$.

\subsubsection{Hedonic and eudaimonic wellbeing}

The Satisfaction with Life Scale (Diener, Emmons, Larsen, \& Griffin, 1985) was used to measure global life satisfaction. Participants indicated the extent to which they agree with five statements on a seven-point Likert scale $(1=$ strongly disagree, to $7=$ strongly agree $)$. Ratings were averaged across items such that higher scores indicated greater satisfaction with life $(\alpha=.86)$. The Scale of Positive and Negative Experience (Diener, Wirtz, Tov, et al., 2010) was used to measure the frequency of an individual's positive and negative affective experiences over the past four weeks. The scale contains six items related to positive feelings and six items related to negative feelings. Answers are indicated by a self-rating on a five-point Likert scale $(1=$ very rarely or never, to $5=$ very often or always). Ratings were averaged, such that higher scores indicated more frequent positive and negative affective experiences respectively ( $\alpha \mathrm{s}=.83$ and .83$)$.

The 18-item Scales of Psychological Wellbeing (SPWB, Ryff, 1989; Ryff \& Keyes 1995) was used as a global measure of six dimensions of psychological wellbeing: autonomy, environmental mastery, personal growth, positive relations with others, purpose in life, and self-acceptance. Responses were indicated on a six-point Likert scale $(1=$ strongly disagree to $6=$ strongly agree $)$ with eight of the items reverse-coded. Ratings were averaged across all 18 items, such that higher scores represented greater psychological wellbeing $(\alpha=.78)$. In addition, the 21-item Questionnaire for Eudaimonic Wellbeing (QEWB, Waterman et al., 2010) was used as a general measure of six categories of eudaimonia: self-discovery, development of one's potential, purpose and meaning, effort in pursuit of excellence, intense engagement, and enjoyment of personally expressive activities. Responses were indicated on a five-point Likert scale $(0=$ strongly disagree to $4=$ strongly agree) with seven of the items reverse-coded. Ratings were averaged across all 21 items such that higher scores indicated greater eudaimonic wellbeing $(\alpha=.78)$.

\section{Results}

Descriptive statistics for the study measures are shown in Table 1 (below).

\subsection{Hedonic and eudaimonic wellbeing}

In a preliminary analysis, confirmatory factor analysis was used to examine the structure of the five wellbeing indicators. A one-factor model was tested in which all five indicators were specified as loading onto a single latent factor (latent factor variance was fixed to 1 ; all loadings were freely estimated). The overall fit for this model was poor $\left(\chi^{2}=76.71, d f=5, p<.001 ; \mathrm{CFI}=\right.$ $.87, \mathrm{SRMR}=.07, \mathrm{RMSEA}=.21$ ) owing to a large residual correlation between the PWB and QEWB scale scores. A second model was tested comprising two correlated latent factors in which life satisfaction, positive affect, and negative affect loaded onto a latent hedonic wellbeing factor, whereas the SPWB and QEWB loaded onto a latent eudaimonic wellbeing factor (variances for each factor were fixed to 1 , loadings on each factor were freely estimated). This model provided excellent fit $\left(\chi^{2}=3.59, d f=4, p=.465 ; \mathrm{CFI}>.99\right.$, SRMR $=.01$, RMSEA $\left.<.01\right)$. Thus, prior to 
subsequent analysis, a composite hedonic wellbeing score was computed by standardizing the life satisfaction, positive affect, and negative affect (reverse-scored) values, then averaging the three measures and standardizing the resulting composite score. A composite eudaimonic wellbeing variable was computed by standardizing the two scale scores, averaging the two measures, and standardizing the resulting composite score.

Table 1. Means, standard deviations, and correlations among study variables

\begin{tabular}{|c|c|c|c|c|c|c|c|c|c|c|c|c|c|}
\hline Variable & $M$ & $S D$ & 1 & 2 & 3 & 4 & 5 & 6 & 7 & 8 & 9 & 10 & 11 \\
\hline 1. General orientation-hedonic & 5.59 & 0.80 & -- & & & & & & & & & & \\
\hline 2. General orientation-eudaimonic & 5.87 & 0.78 & $.44^{*}$ & -- & & & & & & & & & \\
\hline 3. Activity motives-hedonic & 5.04 & 1.09 & $.47^{*}$ & .08 & -- & & & & & & & & \\
\hline 4. Activity motives-eudaimonic & 4.79 & 1.19 & $.15^{*}$ & $.41^{*}$ & $.19^{*}$ & -- & & & & & & & \\
\hline 5. Satisfaction with life & 4.33 & 1.19 & .05 & $.14^{*}$ & $.16^{*}$ & $.25^{*}$ & -- & & & & & & \\
\hline 6. Positive affect & 3.78 & 0.57 & $.19^{*}$ & $.26^{*}$ & $.21^{*}$ & $.24^{*}$ & $.55^{*}$ & -- & & & & & \\
\hline 7. Negative affect & 2.65 & 0.73 & -.05 & $-.12^{*}$ & -.04 & $-.12^{*}$ & $-.47^{*}$ & $-.59^{*}$ & -- & & & & \\
\hline 8. SPWB & 4.47 & 0.56 & $.17^{*}$ & $.43^{*}$ & .06 & $.29^{*}$ & $.47^{*}$ & $.50^{*}$ & $-.45^{*}$ & -- & & & \\
\hline 9. QEWB & 2.65 & 0.43 & $.14^{*}$ & $.45^{*}$ & -.04 & $.43^{*}$ & $.35^{*}$ & $.39^{*}$ & $-.31^{*}$ & $.64^{*}$ & -- & & \\
\hline 10. Composite HWB & 0.00 & 1.00 & $.12^{*}$ & $.21^{*}$ & $.17^{*}$ & $.25^{*}$ & $.81^{*}$ & $.86^{*}$ & $-.82^{*}$ & $.57^{*}$ & $.42^{*}$ & -- & \\
\hline 11. Composite EWB & 0.00 & 1.00 & $.17^{*}$ & $.48^{*}$ & .03 & $.40^{*}$ & $.45^{*}$ & $.49^{*}$ & $-.42^{*}$ & $.91^{*}$ & $.91^{*}$ & $.54^{*}$ & -- \\
\hline
\end{tabular}

Note. $N=325$. SPWB $=$ Scales of Psychological Wellbeing score. $\mathrm{QEWB}=$ Questionnaire of Eudaimonic Wellbeing score. $\mathrm{HWB}=$ hedonic wellbeing. $\mathrm{EWB}=$ eudaimonic wellbeing. ${ }^{*} p<.05$.

\subsection{Correlations between hedonia and eudaimonia}

Positive correlations were observed among the three aspects of hedonia (general orientations, motives for daily activities, and wellbeing); these correlations were stronger between general orientations and daily motives than between either of these scores and wellbeing. Positive correlations were also observed among the three aspects of eudaimonia, with comparable magnitudes among general orientations, daily motives, and wellbeing. Positive correlations also were observed between each corresponding pair of hedonic/eudaimonic concepts. The strongest correlation was found between hedonic and eudaimonic wellbeing; the weakest association was found between hedonic and eudaimonic motives for daily activities.

\subsection{General orientations and motives for daily activities predicting wellbeing}

Consistent with predictions, hedonic wellbeing was positively correlated with each of the general orientation and motives for recent activities measures; eudaimonic wellbeing was also positively correlated with each of these variables except hedonic motives for daily activities. To evaluate the combined and unique predictive effects of the general orientation and motives for daily activities scores on each form of wellbeing, we ran two multiple regression models. In these models, hedonic and eudaimonic wellbeing scores were regressed simultaneously onto the 
hedonic and eudaimonic general orientation and motives for daily activities. Results are shown in Table 2. The regression model explained $9 \%$ of the variance in hedonic wellbeing $(R=.30, p<$ .001), and three of the four predictors were uniquely significant, in that higher hedonic wellbeing was uniquely predicted by stronger eudaimonic orientation, as well as stronger hedonic and eudaimonic motives for daily activities. The regression model explained $29 \%$ of the variance in eudaimonic wellbeing $(R=.54, p<.001)$, and two of the four predictors were significant, in that higher eudaimonic wellbeing was uniquely predicted by stronger eudaimonic general orientations and eudaimonic motives for daily activities.

Table 2. Regression of hedonic and eudaimonic wellbeing on hedonic and eudaimonic general orientations and motives for recent activities

\begin{tabular}{|c|c|c|c|c|c|c|c|c|}
\hline \multirow[b]{2}{*}{ Predictor } & \multicolumn{4}{|c|}{ Hedonic wellbeing } & \multicolumn{4}{|c|}{ Eudaimonic wellbeing } \\
\hline & $b$ & SE & $p$ & $\beta$ & $b$ & SE & $p$ & $\beta$ \\
\hline General orientation-hedonic & -.04 & .09 & .614 & -.04 & -.03 & .08 & .713 & -.02 \\
\hline General orientation-eudaimonic & 19 & .09 & .028 & .15 & .51 & .08 & $<.001$ & .40 \\
\hline Activity motives-hedonic & 13 & .06 & .028 & .14 & -.04 & .05 & .476 & -.04 \\
\hline Activity motives-eudaimonic & .14 & .05 & .006 & 17 & .21 & .04 & $<.001$ & .25 \\
\hline
\end{tabular}

Note. $N=325$. Unstandardized (b) regression coefficients, standard errors (SE), $p$ values, and standardized ( $\beta$ ) regression coefficients are shown by predictor (row variable) for hedonic and eudaimonic wellbeing (column variable).

\subsection{Testing the full life hypothesis}

For each participant, the number of hedonic and eudaimonic general orientation and motives for daily activity scores falling into the top $33 \%$ of each distribution was counted. This 'full life' count (ranging from 0 to 4 ) was treated as a categorical independent variable in two between-subjects ANOVAs with composite hedonic and eudaimonic wellbeing scores as dependent variables. Main effects were significant for both hedonic wellbeing $\left(F(4,320)=6.75, p<.001, \eta^{2}=.08\right)$ and eudaimonic wellbeing $\left(F(4,320)=15.06, p<.001, \eta^{2}=.16\right)$. As shown in Figure 1 (below), higher levels of hedonic and eudaimonic wellbeing were found among individuals characterized by high levels of two or more of the hedonic and eudaimonic general orientations and motives for daily activities. Follow-up pairwise comparisons indicated that individuals with a full life count of 2,3, or 4 were characterized by significantly higher wellbeing than the other two groups. At the other extreme, the lowest levels of wellbeing were found among individuals not characterized by high levels on any of the hedonic and eudaimonic general orientations and motives for daily activities. Follow-up pairwise comparisons indicated that individuals with a full life count of 0 were characterized by significantly lower wellbeing than each of the other groups.

\section{Discussion}

\subsection{Associations between hedonia and eudaimonia}

Within hedonic and eudaimonic concepts, general orientations and motives for recent activities were positively but moderately correlated, suggesting that individuals who oriented their lives 
Figure 1. Standardized mean levels of wellbeing (y-axis) by full life count (x-axis) for hedonic (Panel A) and eudaimonic (Panel B) wellbeing.
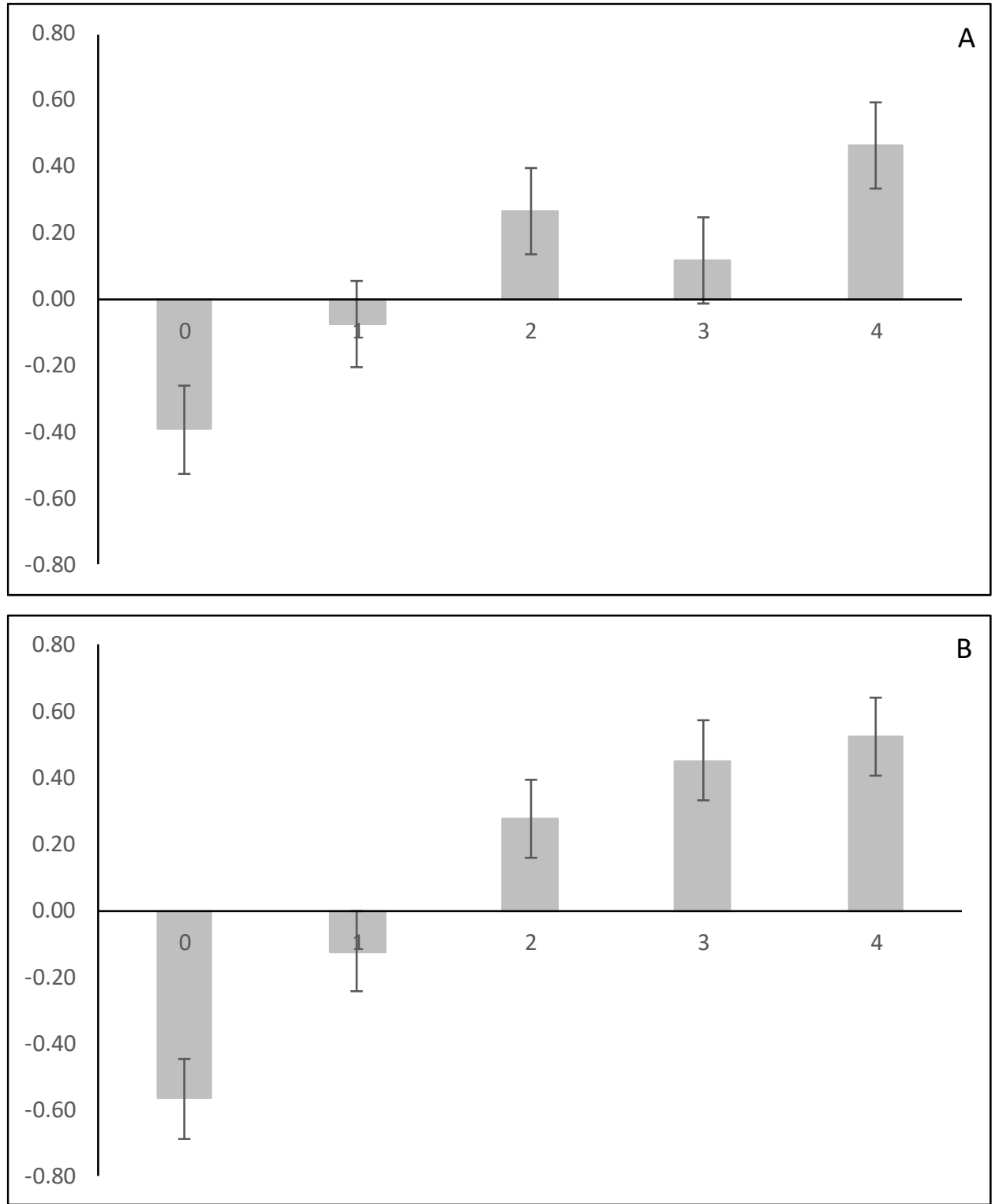

Note. $95 \%$ confidence intervals are shown for each mean. $n \mathrm{~s}=84,83,85,49$, and 24 , respectively, for full life counts of $0,1,2,3$, and 4 .

toward generally seeking pleasure (for example) tended to report recently pursuing daily activities for similar reasons. Although such findings may have resulted from the use of the same set of items to assess general orientation and motives for activities, these results may also suggest that hedonic and eudaimonic motives for daily activities provide some insight into individuals' general orientations (as hypothesized by Huta \& Ryan, 2010). Yet such correspondence was not very strong, indicating that at least some individuals were engaging in recent daily activities for reasons that were inconsistent with their hedonic or eudaimonic orientations. These patterns are consonant with the separation between general motivation orientations and motive states proposed in other motivation research (Carver \& White, 1994; Deci \& Ryan, 2008; Elliot \& Thrash, 2010). The present findings thus support the value of examining general orientations and motives for daily activities as unique aspects of hedonia and eudaimonia. 
Also noteworthy, associations between hedonia and eudaimonia were generally stronger for wellbeing than between motives for recent activities. Such findings provide further indication that the separation between hedonia and eudaimonia may be clearer with respect to motives for daily activities than for experiences of wellbeing (Huta \& Waterman, 2014). Yet there was also evidence of an empirical distinction between hedonic and eudaimonic wellbeing. Specifically, measures of the three components of subjective wellbeing (life satisfaction, positive affect, negative affect) loaded strongly onto a latent factor which was correlated with, but separate from the two scales measuring various aspects of eudaimonic wellbeing. These findings indicate that the five wellbeing measures were not reducible to a single factor and thus support the separability of hedonic and eudaimonic wellbeing, an issue which continues to be debated (e.g., Disabato et al., 2016; Huta, 2016).

\subsection{Predicting hedonic and eudaimonic wellbeing}

Further informing this debate, the present findings provide novel insights concerning similarities and differences between the two types of wellbeing based on their associations with hedonic and eudaimonic orientations and motivations for activities. Importantly, the relative independence of hedonic and eudaimonic general orientations and motives for daily activities raises the possibility that they may have unique psychological significance. Noteworthy in this regard were results from the regression models predicting hedonic and eudaimonic wellbeing. Higher levels of both types of wellbeing were uniquely predicted by the degree to which individuals reported approaching their lives in eudaimonic ways (i.e., seeking excellence and authenticity) and engaging in recent daily activities for eudaimonic reasons. In addition, greater hedonic wellbeing was uniquely predicted by the degree to which individuals reported seeking enjoyment from their recent daily activities. Previous research has demonstrated unique links between hedonic and eudaimonic motives or activities with corresponding experiences of wellbeing (e.g., McGregor \& Little, 1998; Steger et al., 2008). The present findings extend this work by revealing aspects of general orientations and motives for activities that have independent connections to each type of wellbeing.

One interpretation is that a stronger eudaimonic orientation to one's life and daily activities are important precursors to a wide range of wellbeing experiences, including positive experiences (e.g., satisfaction and enjoyment of one's life) and positive functioning (e.g., authenticity, excellence, and meaning). Hedonic motives for daily activities, in contrast, may set the stage for more immediate hedonic wellbeing experiences, but have little unique bearing on an enhanced sense of purpose and self-expressiveness (Huta \& Ryan, 2010). Furthermore, hedonic general orientation was not uniquely predictive of either form of wellbeing. Such findings may suggest that of the two general orientations, generally seeking pleasure and enjoyment in one's life is not uniquely conducive to wellbeing.

Yet other research has indicated unique links between both hedonic and eudaimonic orientations to life and various indicators of wellbeing (e.g., Peterson et al., 2005; Schueller \& Seligman, 2010). No previous studies have examined hedonic and eudaimonic general orientations and motives for activities as joint predictors in the same study. Consequently, the present findings might suggest that a hedonic orientation may simply be the most redundant of the various orientations and motives for activities that we examined. Differences in the unique predictors of hedonic and eudaimonic wellbeing might also reflect an asymmetry in the measurement of wellbeing. Specifically, hedonic wellbeing indicators are more heavily-valenced cognitive-affective reactions to one's life than the indicators of eudaimonic wellbeing, which primarily reflect experiences of flourishing and positive ways of functioning (Huta \& Waterman, 
2014). Further research is needed to examine these possibilities before conclusions can be drawn concerning the unique predictive associations involving the individual aspects of hedonia or eudaimonia.

\subsection{The full life}

Various facets of hedonia and eudaimonic may also be conceptualized as complementary, rather than opposing or competing, parts of a broader system of wellbeing (Huta \& Waterman, 2014; Ryan \& Huta, 2009). Such a system may function to allow individuals to view their lives as manageable (if not enjoyable) and meaningful, even in the face of adversity and threat (Shmotkin, 2005). Previous studies examining the full life hypothesis have found the highest levels of wellbeing among individuals characterized by higher levels of hedonic and eudaimonic orientations to their lives (Peterson et al., 2005; Schueller \& Seligman, 2010) or above-average levels of both hedonic and eudaimonic motives for activities (Huta \& Ryan, 2010; Kryza-Lacombe et al., 2019).

The present study extends this research by showing that the highest (vs. lowest) levels of hedonic and eudaimonic wellbeing were found in individuals characterized by high (top 33\%) levels of all four (rather than no) types of general orientation and motives for recent activities. Seeking both hedonia and eudaimonia in general and through one's daily activities, therefore, may promote a wider range of positive outcomes (including positive experiences, feelings, and functioning) than orienting one's life and daily activities toward either pleasure and satisfaction or authenticity and meaning. We speculate that such complementarity has benefits in terms of the psychological coherence experienced when one's motives for daily activities are congruent with core aspects of the self, including one's general orientation to life (Huta \& Waterman, 2014; McGregor \& Little, 1998).

Nonetheless, comparably high levels of hedonic and eudaimonic wellbeing were also found among individuals characterized by high levels of at least two of the general orientations and activity motives. Such findings suggest that positive wellbeing outcomes may require only, or be supported by even a 'partially full', rather than a completely full life - a finding that may have practical significance given that it likely represents a more realistic goal for many individuals. Future studies should seek to replicate this particular finding, however, and also further inform the significance of various combinations of hedonic and eudaimonic general orientations and activity motives.

\subsection{Limitations and future directions}

In addition to the caveats discussed above, we note that future research is needed to examine the reliability and generalizability of our findings beyond the present sample of younger (primarily female) adults from Canada. Additional testing with more diverse samples of participants, including with respect to age, health status, and other sociodemographic factors relevant to hedonic and eudaimonic orientations, motives, and wellbeing would be valuable. Given differences between western and eastern conceptualizations of happiness and wellbeing (Joshanloo, 2014), cross-cultural testing is also critical.

Also noteworthy, the present predictive results did not inform the underlying mechanism(s). One possibility is that general orientations and motives for daily activities are connected to wellbeing via the types of daily activities in which one engages and resulting experiences of hedonia and eudaimonia in such activities (e.g., Henderson, Knight, \& Richardson, 2013; Steger et al., 2008). Such activities may also lead to greater hedonic and eudaimonic wellbeing by satisfying basic psychological needs (Ryan \& Deci, 2001). Further studies are needed to assess 
such potential mediating factors. We further note that such interpretations assume a causal direction flowing from general orientation and motives for daily activities to wellbeing. Additional research employing a longitudinal design (e.g., Huta \& Ryan, 2010) is needed to test these dynamics.

We also note that some evidence suggests that hedonic and eudaimonic motives may comprise three, rather than two, factors (Asano et al., 2018; Bujacz et al., 2014). In future research it may thus be valuable to consider whether such distinctions are also relevant to general orientations and motives for recent daily activities, and their connections to wellbeing. Furthermore, the motives scale from which the measures employed in the present work were modified (i.e., Huta \& Ryan, 2010) does not assess the motivation to avoid negative outcomes (e.g., pain, distress). As a result, the extent to which this scale fully captures the key components of hedonia (Huta \& Waterman, 2014) is unclear. Further work is needed, therefore, to determine how results concerning the issues addressed in the present work may differ if hedonic orientations and motives for activities are assessed in terms of seeking pleasure and avoiding pain.

In addition, because the general orientations and motives for daily activities scales employed the same set of items, it may have artificially inflated the correlation between corresponding hedonic and eudaimonic scale scores. In future research, it would be valuable to use an alternative measure of general orientation such as the Orientations to Happiness measure (Peterson et al., 2005) in order to provide additional information concerning the associations between hedonic and eudaimonic general orientation and motives for activities.

Finally, beyond the theoretical and scientific implications of the present findings, practical relevance also needs to be tested. In particular, the applied meaningfulness of the distinctions examined in the present work (i.e., hedonia vs. eudaimonia with respect to general orientations, motives for daily activities, experiences of wellbeing; pursuit of one versus multiple types of hedonic and eudaimonic activities) needs to be determined though, for example, intervention studies and qualitative analysis aimed at informing the implications of the present findings for the lives of individuals.

\section{Summary and conclusions}

Hedonic and eudaimonic wellbeing were identified as related but distinct factors. General orientations and motives for recent activities were related but separable, both within and between hedonic and eudaimonic concepts. Eudaimonic (more so than hedonic) general orientations and motives for daily activities were uniquely associated with both forms of wellbeing. Yet, consistent with the full life notion, both hedonic and eudaimonic wellbeing were highest among individuals characterized by high levels of hedonic and eudaimonic general orientations and motives for daily activities. We conclude, therefore, that studying hedonia and eudaimonia as related but distinct concepts provide valuable new insights, not only with respect to experiences of wellbeing, but also in terms of individuals' general orientations to their lives and motives for their daily activities.

\section{Acknowledgements}

This work was supported by the Social Sciences and Humanities Research Council of Canada.

\section{Conflict of interest statement}

The authors report no conflicts of interest. 


\section{Authors}

Taysa-Rhea Mise

Brock University

Michael A. Busseri

Brock University

mbusseri@brocku.ca

\section{Publishing Timeline}

Received 17 July 2019

Revised submission received 18 March 2020

Accepted 18 August 2020

Published 30 September 2020

\section{References}

Asano, R., Tsukamoto, S., Igarashi, T., \& Huta, V. (2018). Psychometric properties of measures of hedonic and eudaimonic orientations in Japan: The HEMA scale. Current Psychology. https://doi.org/10.1007/s12144-018-9954-z

Bujacz, A., Vitterso, J., Huta, V., \& Kaczmarek, L. D. (2014). Measuring hedonia and eudaimonia as motives for activities: Cross-national investigation through traditional and Bayesian structural equation modeling. Frontiers in Psychology, 5, 1-10. https://doi.org/10.3389/fpsyg.2014.00984

Busseri, M. A., \& Sadava, S. W. (2011). A review of the tripartite structure of subjective well-being: Implications for conceptualization, operationalization, analysis, and synthesis. Personality and Social Psychology Review, 15(3), 290-314. https://doi.org/10.1177/1088868310391271

Carver, C. S., \& White, T. L. (1994). Behavioral inhibition, behavioral activation, and affective responses to impending reward and punishment: The BIS/BAS scales. Journal of Personality and Social Psychology, 67(2), 319-333. https://doi.org/10.1037/0022-3514.67.2.319

Deci, E. L. \& Ryan, R. M. (2008). Facilitating optimal motivation and psychological well-being across life's domains. Canadian Psychology, 49(1), 14-23. https://doi.org/10.1037/0708-5591.49.1.14

Diener, E. (1984). Subjective well-being. Psychological Bulletin, 95, 542-575. https://doi.org/10.1037/00332909.95.3.542

Diener, E., \& Lucas, R. E. (1999). Personality and subjective well-being. In D. Kahneman, E. Diener, \& N. Schwartz (Eds), Well-being: The foundations of hedonic psychology (pp. 213-229). New York, NY: Russell Sage Foundation.

Diener, E., Emmons, R., A., Larsen, R., J., \& Griffin, S. (1985). The satisfaction with life Scale. Journal of Personality Assessment, 49(1), 71- 75. https://doi.org/10.1207/s15327752jpa4901_13

Diener, E., Wirtz, D., Tov, W., Kim-Prieto, C., Choi. D., Oishi, S., \& Biswas-Diener, R. (2010). New measures of well-being: Short scales to assess flourishing and positive and negative feelings. Social Indicators Research, 97(2), 143-156. https://doi.org/10.1007/s11205-009-9493-y

Disabato, D. J., Goodman, F. R., Kashdan, T. B., Short, J. L., \& Jarden, A. (2016). Different types of wellbeing? A cross-cultural examination of hedonic and eudaimonic well-being. Psychological Assessment, 28, 471-482. https://doi.org/10.1037/pas0000209

Elliot, A. J., \& Thrash, T. M. (2002). Approach-avoidance motivation in personality: Approach and avoidance temperaments and goals. Journal of Personality and Social Psychology, 82(5), 804-818. https://doi.org/10.1037/0022-3514.82.5.804

Gallagher, M. W., Lopez, S. J., \& Preacher, K. J. (2009). The hierarchical structure of well-being. Journal of Personality, 77, 1025-1050. https://doi.org/10.1111/j.1467-6494.2009.00573.x

Henderson, L. W., Knight, T., \& Richardson, B. (2013). An exploration of the well-being benefits of hedonic and eudaimonic behavior. Journal of Positive Psychology, 8, 322-336.

https://doi.org/10.1080/17439760.2013.803596 
Huta, V. (2013). Pursuing eudaimonia versus hedonia: Distinctions, similarities, and relationships. In A. Waterman (Ed.). The Best Within Us: Positive Psychology Perspectives on Eudaimonia. (pp. 139-158). American Psychological Association: Washington DC. https://doi.org/10.1037/14092-008

Huta, V. (2016). Eudaimonic and hedonic orientations: Theoretical considerations and research findings. In J. Vitterso (Ed.). Handbook of Eudaimonic Well-Being. (pp. 215-231). Springer: Switzerland. https://doi.org/10.1007/978-3-319-42445-3_15

Huta, V., Pelletier, L. G., Baxtor, D., \& Thompson, A. (2012). How eudaimonic and hedonic motives related to the well-being of close others. Journal of Positive Psychology, 7, 399-404. https://doi.org/10.1080/17439760.2012.705318

Huta, V. \& Ryan, R. M. (2010). Pursuing pleasure or virtue: The differential and overlapping well-being benefits of hedonic and eudaimonic motives. Journal of Happiness Studies, 11, 735-762. https://doi.org/10.1007/s10902-009-9171-4

Huta, V. \& Waterman, A. (2014). Eudaimonia and its distinction from hedonia: Developing a classification and terminology for understanding conceptual and operational definitions. Journal of Happiness Studies, 15, 1425-1456. https://doi.org/10.1007/s10902-013-9485-0

Joshanloo, M. (2014). Eastern conceptualizations of happiness: Fundamental differences with western views. Journal of Happiness Studies, 15, 475-579. https://doi.org/10.1007/s10902-013-9431-1

Joshanloo, M. (2016). Revisiting the empirical distinction between hedonic and eudaimonic aspects of well-being using exploratory structural equation modeling. Journal of Happiness Studies, 17, 20232036. https://doi.org/10.1007/s10902-015-9683-z

Kashdan, T. B., Biswas-Diener, R., \& King, L. A. (2008). Reconsidering happiness: The costs of distinguishing between hedonics and eudaimonia. Journal of Positive Psychology, 3, 219-233. https://doi.org/10.1080/17439760802303044

Keyes, C. L. M., Ryff, C. D., \& Shmotkin, D. (2002). Optimizing well-being: The empirical encounter of two traditions. Journal of Personality and Social Psychology, 82(6), 1007-1022. https://doi.org/10.1037/0022-3514.82.6.1007

Kryza-Lacombe, M., Tanzini, E., \& O’Neill, S. (2019). Hedonic and eudaimonic motives: Associations with academic achievement and negative emotional states among urban college students. Journal of Happiness Studies, 20, 1323-1341. https://doi.org/10.1007/s10902-018-9994-y

McGregor, I., \& Little, B. R. (1998). Personal projects, happiness, and meaning: On doing well and being yourself. Journal of Personality and Social Psychology, 76, 494-512. https://doi.org/10.1037/00223514.74.2.494

Peterson, C. Park, N., \& Seligman, M. E. P. (2005). Orientations to happiness and life satisfaction: The full life versus the empty life. Journal of Happiness Studies, 6, 25-41. https://doi.org/10.1007/s10902-0041278-z

Ryan, R. M., \& Deci, E. L. (2001). On happiness and human potentials: A review of research on hedonic and eudaimonic well-being. Annual Review of Psychology, 52, 141-166. https://doi.org/10.1146/annurev.psych.52.1.141

Ryan, R. M., Huta, V., \& Deci, E. L. (2008). Living well: A self-determination theory perspective on eudaimonia. Journal of Happiness Studies, 9(1), 139-170. https://doi.org/10.1007/s10902-006-9023-4

Ryff, C. D. (1989). Happiness is everything, or is it? Explorations on the meaning of psychological wellbeing. Journal of Personality and Social Psychology, 57, 1069-1081. https://doi.org/10.1037/00223514.57.6.1069

Ryff, C., \& Keyes, C. (1995). The structure of psychological well-being revisited. Journal of Personality and Social Psychology, 69, 719-727. https://doi.org/10.1037/0022-3514.69.4.719

Schueller, S. M., \& Seligman, M. E. P. (2010). Pursuit of pleasure, engagement, and meaning: Relationships to subjective and objective measures of well-being. Journal of Positive Psychology, 5, 253264. https://doi.org/10.1080/17439761003794130

Seligman, M. E. P. (2002). Authentic happiness. Free Press: New York, NY.

Shmotkin, D. (2005). Happiness in the face of adversity: Reformulating the dynamic and modular bases of subjective well-being. Review of General Psychology, 9, 291-325. https://doi.org/10.1037/1089-

2680.9.4.291 
Steger, M. F., Kashdan, T. B., \& Oishi, S. (2008). Being good by doing good: Daily eudaimonic activity and well-being. Journal of Research in Personality, 42, 22-42. https://doi.org/10.1016/j.jrp.2007.03.004

Waterman, A. S. (1993). Two conceptions of happiness: Contrasts of personal expressiveness (eudaimonia) and hedonic enjoyment. Journal of Personality and Social Psychology, 64, 678-691. https://doi.org/10.1037/0022-3514.64.4.678

Waterman, A. S., Schwartz, S. J., \& Conti, R. (2008). The implications of two conceptions of happiness (hedonic enjoyment and eudaimonia) for the understanding of intrinsic motivation. Journal of Happiness Studies, 9, 41-79. https://doi.org/10.1007/s10902-006-9020-7

Waterman, A. S., Schwartz, S. J., Zamboanga, B. L., Ravert, R. D., Williams, M. K., Agocha, V. B., Kim, S. Y., \& Donnellan, M. B. (2010). The questionnaire for eudaimonic well-being: Psychometric properties, demographic comparisons, and evidence of validity. Journal of Positive Psychology, 5, 4161. https://doi.org/10.1080/17439760903435208 